\title{
MITTEILUNGEN
}

\section{„Parteien und Demokratie - Innerparteiliche Demokratie im Wandel“". Fachwissenschaftliche Tagung in der Akademie für Politische Bildung Tutzing vom 16. bis 18. März 2012}

Sie leben, die Parteien. Das stellte einmal mehr die Tutzinger Tagung zur innerparteilichen Demokratie in Deutschland und in ausgewählten westlichen Demokratien unter Beweis. Das für den Freitagabend geplante Akademiegespräch am See zum „Geheimnis in der Demokratie“, zu dem der Tübinger Philosoph Otfried Höffe und Justizministerin Sabine Leutheusser-Schnarrenberger eingeladen waren, musste leider aufgrund der aktuellen Ereignisse der Bundespräsidentenwahl auf einen späteren Termin verschoben werden. Stattdessen ging der Tagung eine Diskussion zum Thema „Liquid Democracy“ voraus. Auf dem Podium nahmen Platz: Martin Delius, Parlamentarischer Geschäftsführer der Piraten im Berliner Abgeordnetenhaus, Reinhard Brandl, MdB (CSU) und Stellvertretender Landesvorsitzender von CSUnet, und Ursula Münch, Direktorin der Akademie und launige Moderatorin des Abends. Zunächst einmal klärte sie die gut 60 Besucher der Abendveranstaltung auf, was diese denn unter „Liquid Democracy“, in wortgetreuer Übersetzung also „flüssige Demokratie“, zu verstehen haben: eine Mischform von direkter und repräsentativer Demokratie, die das Ziel verfolgt, eine breitere Beteiligung der Bürger an der Parteiendemokratie zu ermöglichen - entweder direkt oder indirekt über Delegierte.

Seit 2009 macht der Begriff „Liquid Democracy“ Karriere in der Öffentlichkeit. Auslöser dafür waren, das gestand Reinhard Brandl ein, die zwei Prozent an Stimmen für die Piratenpartei bei der Bundestagswahl 2009. Im selben Jahr gründete sich der - nicht mit der Piratenpartei zu verwechselnde - überparteiliche und gemeinnützige Verein „Liquid Democracy e.V.“ (http://liqd.net/), der seine speziell entwickelte Software „Adhocracy“ interessierten Anwendern - Parteien, Interessengruppen, der Wissenschaft - zur Verfügung stellt. Neben dem SPD-Bundesvorstand arbeitet auch die im Mai 2010 eingerichtete Enquete-Kommission „Internet und digitale Gesellschaft“ des Deutschen Bundestages (http://www.bundestag. de/internetenquete/) mit dieser Software, um „Diskussionen, Vorschläge und Ideen“ der Bürger in ihre Arbeit einfließen zu lassen (https://enquetebeteiligung.de/). Sowohl Delius als auch Brandl kritisierten, dass diese Beteiligungsplattform von der Enquete-Kommission zunächst nicht ihren Möglichkeiten entsprechend genutzt wurde. Beide bekannten sich als große Fans der digitalen Beteiligungsmöglichkeiten und lobten die Expertise, die sich auf diesem Wege einholen lasse. Beide betonten aber auch, dass „Liquid Democracy“ kein Ersatz für repräsentative Demokratie sein könne. Hier waren die Positionen des CSU-Abgeordneten und des Vertreters der Piratenpartei bemerkenswert nahe beieinander. Erstaunen beim Publikum, bei manch einem wohl auch Skepsis, rief das klare Bekenntnis des Piraten zu seinem Selbstverständnis als Repräsentant, der unabhängig vom eingeholten Votum der Netzgemeinde entscheiden müsse, hervor. Das unverblümte Bekenntnis Delius', Elite zu sein, das mit dem Anspruch kollidiere, eine Partei flacher Hierarchien sein zu wollen, klang fast ein bisschen zu abgebrüht. Hier offenbarte sich, dass der Vergleich mit den Grünen, der an diesem Abend oft gezogen wurde, im Hinblick auf das Demokratieverständnis eine Menge Fragen aufwirft.

Am Samstagmorgen eröffnete Elmar Wiesendahl aus Hamburg die wissenschaftliche Fachdiskussion mit einem sehr lebhaften, charismatischen Vortrag zum Thema „Michels' ehernes 
Gesetz der Oligarchie - Bleibendes und Revisionsbedürftiges nach hundert Jahren“. Tenor seines Beitrags war die Behauptung, dass die Sozialwissenschaften über Robert Michels' Hauptwerk „Zur Soziologie des Parteiwesens in der modernen Demokratie“ im Kern bis heute nicht hinaus gelangt seien. Zwar sei die Zeit empirisch über Michels hinweggegangen, es bleibe aber das immer wiederkehrende Problem der Abkoppelung der Parteiführer von der Basis. Elitenherrschaft aus der Welt zu schaffen, so die Quintessenz des Vortrags, bleibe deshalb notwendig eine Illusion. Gelingen könne allenfalls die Herrschaftsbegrenzung der Gewählten über die Wähler. Die sei im Grunde ein permanenter Auftrag an das Ethos des Bürgers, sich beim allmorgendlichen Blick in den Spiegel als selbstbestimmtes Individuum und nicht als Teil einer apathischen, entpolitisierten Masse im Sinne Gustave Le Bons sehen zu wollen. Ein weiser Satz, der als Fußnote zur Podiumsdiskussion des vorigen Abends gelesen werden konnte, aber auch die theoretische Hintergrundfolie für die kommenden Vorträge bot.

Im Anschluss stellte Eike-Christian Hornig (Technische Universität Darmstadt) sein Forschungsprojekt über „Konzepte vertikaler Parteistrukturen im Wandel stadientypologischer Modelle"vor. Hier soll es um die Untersuchung des Zusammenhangs von Regionalisierung, Föderalisierung, Territorialität einerseits und den Parteistrukturen andererseits gehen. Ausgangshypothese ist die Beobachtung, dass die in der Parteienforschung gebräuchliche Stadientypologie, die eine fortschreitende Entwicklung von der Honoratioren- über die Massenintegrations- und Volkspartei hin zur Partei der Berufspolitiker - auch der Kartellpartei oder der „Franchise Party“ - ausgemacht hat, erstaunlich wenig über die vertikalen Parteistrukturen aussagt.

Im zweiten inhaltlichen Block des Vormittags stieg Margret Hornsteiner aus den luftigen Höhen der Theorie hinab in die empirischen Niederungen der deutschen Parteiendemokratie. In ihrem systematisch klar strukturierten, an der „Prinzipal-Agent“-Theorie ausgerichteten Vortrag über den „Entstehungsprozess von Wahlprogrammen“ ging Hornsteiner, Doktorandin bei Thomas Saalfeld in Bamberg, der Frage nach, ob unterschiedliche „Varianten der innerparteilichen Demokratie“ bei SPD und Grünen Folgen für den Charakter ihrer Wahlprogramme haben. Mit Blick auf den Erstellungsprozess der Bundestagswahlprogramme 1998 kam sie zu dem Ergebnis, dass das Mehr an innerparteilicher Demokratie bei den Grünen zu einem konkreteren Wahlprogramm geführt habe als bei der SPD, die zwar ihren Mitgliedern einen längeren Vorlaufprozess für die Programmarbeit zugestand, ihnen am Ende aber die Programmformulierung aus der Hand nahm und auch (anders als die Grünen) keine Änderungsanträge am Entwurf mehr zuließ. Das Ergebnis sei ein weit weniger detailliertes Programm mit weniger konkreten Wahlversprechen gewesen.

Auch Deniz Anan (Technische Universität München) stellte messbare Varianten innerparteilicher Demokratie fest - diesmal im Vergleich von FDP und Grünen. Er untersuchte die „Repräsentations- und Entscheidungskulturen auf Parteitagen“ und kam zu dem Befund, dass sich diese doch merklich unterschieden. Anan stellte eine intime Kenntnis der Satzungen beider Parteien unter Beweis, wie sie gelegentlich unter Parteienforschern heute vermisst wird. So konnte er unter anderem zeigen, dass die Grünen entgegen einem anders lautenden Vorurteil auch nach 30 Jahren noch keine Partei wie jede andere geworden sind. Es gibt nach wie vor diverse Quotenregelungen; es gibt die „Doppelspitze“ in der Führung von Partei und Fraktion; es gibt - im Bund und von Landesverband zu Landesverband unterschiedliche Regelungen hinsichtlich der Trennung von Amt und Mandat; und es gibt weitreichende auch finanzielle - Absicherungen der innerparteilichen Mitgliederbeteiligung und Mitbestimmung. Repräsentanten der Grünen verfügen insgesamt über deutlich weniger 
Handlungsspielräume als diejenigen der FDP, müssen sich weit stärker gegenüber ihrer innerparteilichen Basis rechtfertigen und werden auch eher zur Rechenschaft gezogen.

Der Samstagnachmittag war dem Feld der innerparteilichen Kandidatennominierung im weitesten Sinne vorbehalten. Klaus Detterbeck (Universität Magdeburg) gab einen kurzen Einblick in die theoretische Debatte und wies auf die eminente Bedeutung der Frage für die innerparteiliche Demokratie hin: Wer wählt den Parteiführer oder den Spitzenkandidaten? Besteht das "Selektorat" nur aus den Parteiführern selbst, handelt es sich mithin um eine Selbstrekrutierung - oder werden Fraktionen, Parteivorstände, Parteitage, Parteimitglieder, möglicherweise gar die Wähler an dieser Auslese beteiligt. Die Abschließung oder Öffnung der Auswahlverfahren hat Folgen für Partizipation, Repräsentation, Parteienwettbewerb und Kandidatenqualität. Die internationale theoretische Debatte kreist im Spannungsfeld von "Circumventing the Activists" (Richard Katz / Peter Mair), wonach eine Demokratisierung durch Urwahlen illusorisch sei und zur weiteren Abschottung der Eliten führe, und „Bringing the Members Back In" (Lawrence LeDuc / Susan Scarrow / André Blais), worin ein Demokratisierungspotential gesehen wird. Empirisch habe sich seit den 1990er Jahren europaweit durchaus das Bild einer Öffnung der Auswahlverfahren ergeben. Mit Blick auf die „Urwahlen in deutschen Landesparteien“ konstatierte Detterbeck zwar auch eine Zunahme, sprach aber doch insgesamt vom „seltenen Glück der Wahl“. Nach wie vor dominierten in den deutschen Bundesländern (wie auch im Bund) die „Krönungswahlen“. Wo es zur Urwahl der Spitzenkandidaten kam, sei ein Bündel von drei Motiven ausschlaggebend gewesen: Parteien wollten den Bekanntheitsgrad ihres Kandidaten steigern und die „elektorale Dividende" steigern; sie wollten Mitglieder mobilisieren; oder sie wollten innerparteiliche Blockaden im Kampf um Führungsämter überwinden.

Die Wahrscheinlichkeit einer Parteireform nimmt zu, so ein Befund der internationalen „Party Change“-Forschung (Robert Harmel / Kenneth Janda), wenn mindestens eines der folgenden drei Ereignisse eintritt: ein Führungswechsel innerhalb der Partei, ein Wechsel dominanter Faktionen oder ein externer Schock. Nach dem Fiasko bei der Bundestagswahl 2009 und der Wahl von Sigmar Gabriel zum Parteivorsitzenden lagen bei der SPD mindestens zwei, wenn nicht gar alle drei Faktoren vor. Dass die neue Parteiführung um Gabriel und Andrea Nables zur Organisationsreform schritt, konnte insofern nicht verwundern, wie Sebastian Bukow (Universität Düsseldorf) überzeugend argumentierte. Ist die SPD aber wirklich, wie sie propagandistisch verkündet, „,[m]it Primaries und verstärkter Basisbeteiligung auf dem Weg zur modernsten Partei Europas?"“ Bukow musste die Frage verneinen: Eine „neue Partizipationshandschrift“ lasse das im Dezember 2011 verabschiedete Organisationspolitische Grundsatzprogramm der SPD nicht erkennen; auch trugen die Delegierten die Reform nur sehr eingeschränkt mit. Das vielleicht aussagekräftigste Ergebnis im internen Vorbereitungsprozess der Reform war die Tatsache, dass sich 86 Prozent der befragten Ortsvereine gegen eine Beteiligung von Nicht-Mitgliedern bei Sachentscheiden, 66 Prozent gegen eine Beteiligung von Nicht-Mitgliedern bei der Kandidatenauswahl für öffentliche Ämter aussprachen. Für Bukow hat die SPD-Parteireform in erster Linie eine „Retraditionalisierung der Partei als Mitgliederpartei“ bewirkt; die Parteireform sei nicht mehr als eine „Mitgliederbeteiligungsermöglichungsreform“ geworden.

Am späten Samstagnachmittag kam der Blick über die deutschen Grenzen hinaus zu seinem Recht: Patrick Horst (Universität Passau) spürte der ,unendlichen Reform des präsidentiellen Nominierungsprozesses in den USA“, dem Mutterland der „Primaries“, nach. Er versuchte nachzuweisen, dass die US-amerikanischen Parteien mit den „Primaries“ - trotz 
vielfältiger Klagen über das „Frontloading“ im Vorwahlkalender, den Wettlauf um Spenden und den Abschreckungseffekt des mindestens zweijährigen Wahlkampfs für die vermeintlich „besten“ Kandidaten - im Prinzip erstaunlich gut gefahren sind. Der nicht selten chaotisch anmutende Prozess der ständigen Reform der Reform habe zwar zu einem Nominierungsverfahren geführt, das die Handlungsfähigkeit der Parteien bis an ihre Grenzen strapaziere; dennoch seien bessere Alternativen nicht in Sicht: Weder die „National Primary“ noch die „Regional Primary“ oder gar die Rückkehr zum nationalen Parteikonvent als faktischem Nominierungsorgan zeigten gangbare Wege auf. Die Rufe nach dem Bundes- oder gar dem Verfassungsgesetzgeber verhüllten nur mehr schlecht als recht, dass es politische Mehrheiten für keinen der zahlreichen Reformvorschläge gebe. Sie seien deshalb, so Horst, bisher zu Recht nicht erhört worden. Am besten führen die Parteien auch weiterhin damit, wenn sie ihre nationalen und einzelstaatlichen Interessen selbst zu einem Ausgleich brächten. Für 2012 gelang Demokraten und Republikanern zur Überraschung manch eines Parteienforschers ein bemerkenswerter Abbau des „Frontloading“. So ausgewogen und balanciert wie in diesem Jahr war der Vorwahlkalender seit langem nicht mehr.

Den Samstag beschlossen Ed Turner (Aston University Birmingham) und Daniel Wigbers (University of Cambridge) mit dem Thema: „Ambitionierte Zielsetzung, unzureichende Realisierung? Reformen der Kandidatenrekrutierung in Großbritannien und Deutschland im Vergleich“. Länderübergreifend waren sie sich darin einig, dass Reformen schwer zu realisieren seien. Hinsichtlich der Praxis der Kandidatenrekrutierung beobachtete Wigbers in Großbritannien eine etwas größere Offenheit gegenüber Quereinsteigern, während Turner für Deutschland doch ein Standardformat des idealen Kandidaten ausmachte: Jurist, Beamter, männlich, lokal verwurzelt, mittleren Alters. Eine mögliche Erklärung sahen beide in dem stärkeren Durchgriff, den die Parteizentralen in Großbritannien auf die Kandidatenaufstellung vor Ort haben. In Deutschland sei demgegenüber die Autonomie der Landesverbände, wenn nicht gar der Kreisverbände deutlich stärker ausgeprägt.

Am Sonntagvormittag stellte Tagungsleiter Henrik Gast (Universität Regensburg) sein Forschungsprogramm „Parteien als oligarchische Organisationen? Innerparteiliche Demokratie im internationalen Vergleich" vor. Hier will er hinterfragen, ob die Renaissance der Michelschen Oligarchiethese in der internationalen Parteienforschung empirisch haltbar ist. Unter dem Titel „Mehr Vernetzung wagen? Zur veränderten Ausrichtung innerparteilicher Kommunikationskanäle und -prozesse der SPD im Social Media-Zeitalter und deren Implikationen für die Party Change-Forschung“ analysierten Isabelle Roth und Daniel Reichard (Universität Trier) die digitale Netzwerkbildung von Untergliederungen der SPD über „Facebook“ und "Twitter" mit dem Ergebnis, dass es keine systematischen Vernetzungen gebe. Abrunden durfte die interessante und lebhafte Tagung Nicolai Dose von der Universität Duisburg-Essen. Er stellte unter dem Thema „Innerparteiliche Demokratie und Mitgliederschwund" eine explorative Studie vor, die den innerparteilichen Ursachen für den Mitgliederrückgang bei der SPD nachspürt. Dabei dürfte es sich um den ersten Versuch handeln, Austrittsmotive systematisch zu erfassen. Ein Ergebnis war, dass das kurzfristige „Durchdrücken" eines Politikwechsels von oben ohne breite innerparteiliche Diskussion zu Mitgliederaustritten führt. Mehr Diskussion in den Parteien und mehr Diskussion über die Parteien, wie sie diese Tagung bot, können den Parteien also nur gut tun. 